\title{
Beispiele asbestfaserstaubbedingter Veränderungen im HRCT - Anhaltspunkte für die Kausalzuordnung bei Vorsorgeuntersuchungen und bei der gutachterlichen Beurteilung
}

\author{
Examples for Asbestos-Related Findings in HRCT - Criteria for the Assessment of Causal Relationships \\ in Surveillance Programmes and Medical Expert Opinion
}

Autoren

Institute
T. Kraus ${ }^{1}$, E. Borsch-Galetke ${ }^{2}$, H. J. Elliehausen ${ }^{3}$, K. Frank ${ }^{4}$, K. G. Hering ${ }^{5}$, H. G. Hieckel ${ }^{6}$, K. Hofmann-Preiß ${ }^{7}$, W. Jacques ${ }^{8}$, U. Jeremie ${ }^{9}$, N. Kotschy-Lang ${ }^{10}$, E. Mannes ${ }^{11}$, H. Otten ${ }^{12}$, W. Raab ${ }^{13}$, H. J. Raithel ${ }^{14}$, W. D. Schneider ${ }^{15}$, S. Tuengerthal ${ }^{16}$

Die Institutsangaben sind am Ende des Beitrags gelistet. eingereicht 16.9.2009

akzeptiert 12.10.2009

\section{Bibliografie}

Dol http://dx.doi.org/ 10.1055/s-0029-1215338

Online-Publikation: 11. 12. 2009

Pneumologie 2010; 64:

37-44 @ Georg Thieme

Verlag KG Stuttgart · New York

ISSN 0934-8387

\section{Korrespondenzadress \\ Univ. Prof. Dr. med. \\ Thomas Kraus}

Institut für Arbeitsmedizin und

Sozialmedizin

Universitätsklinikum,

RWTH Aachen University

Pauwelsstraße 30

52074 Aachen

tkraus@ukaachen.de

www.arbeitsmedizin.ukaachen.de

\section{Zusammenfassung \\ $\nabla$}

In der Diagnostik asbestbedingter Erkrankungen wird immer häufiger die Computertomografie in Hochauflösungstechnik (HRCT) eingesetzt. Für eine exakte Diagnosestellung bedarf es genauer Kriterien, wann ein Kausalzusammenhang zwischen bildmorphologischem Befund und früherer Asbestexposition angenommen werden kann. Dies ist sowohl für arbeitsmedizinische Vorsorgeuntersuchungen als auch für die gutachterliche Kausalzusammenhangsbeurteilung von Bedeutung. Die vorliegende Arbeit gibt Beispiele für asbestbedingte parenchymale und pleurale Veränderungen und diskutiert die Spezifität dieser Befunde.

\section{Einleitung}

$\nabla$

Der zunehmende Einsatz der hochauflösenden Computertomografie (HRCT) in der Begutachtung asbestfaserstaubbedingter Erkrankungen von Pneumokoniosen und in Vorsorgeuntersuchungen nach dem berufsgenossenschaftlichen Grundsatz G1.2 erfordert eine subtile Interpretation und differenzialdiagnostische Abgrenzung röntgenmorphologischer Befunde. Durch die hohe Sensitivität der HRCT werden Veränderungen an Lunge und/oder Pleura dargestellt, die vor allem in ihren frühen Stadien nicht immer unmittelbar kausal zuzuordnen sind und im Vergleich zur Beurteilung konventioneller Röntgenaufnahmen profunde differenzialdiagnostische Überlegungen erforderlich machen. Die Arbeitsgruppe, die die internationale CT-Klassifikation erarbeitet hat, hat mittlerweile auch Referenzfilme analog zu den Standardfilmen der ILO-Klassifikation ausgewählt und validiert [1]. Dies erleichtert die Quantifizierung und ätiologische Zuordnung der Befunde. Problematisch bei der Interpretation von HRCT-Befunden bleibt aber, dass bisher nur

\section{Abstract \\ $\nabla$}

The increasing use of high-resolution computed tomography in formerly asbestos-exposed workers requires valid diagnostic criteria for the findings which have to be reported as suspicious for being asbestos-related in surveillance programmes and for the assessment of causal relationships between former asbestos exposure and findings in computed tomography. The present article gives examples for asbestos-related findings in HR-CT and discusses the specificity of parenchymal and pleural changes due to asbestos fibres.

begrenzt Daten zur Prävalenz asbestbedingter Veränderungen vorliegen, während die Datenlage für konventionelle Röntgenbilder zurzeit noch besser ist.

Ziel dieser Arbeit ist es daher, durch beispielhafte Bildmuster im HRCT eine Hilfestellung bei der Interpretation der kausalen Zuordnung parenchymaler und pleuraler Veränderungen sowohl bei Vorsorgeuntersuchungen als auch bei der Begutachtung zu liefern.

\section{Parenchymale Veränderungen nach Asbestexposition}

Röntgenmorphologisch können sich Veränderungen nach Asbestfaserstaubexposition als irreguläre intralobuläre - und/oder als interlobuläre Schatten bzw. als sog. Parenchymbänder manifestieren. Diese Veränderungen sind jedoch ätiologisch zunächst vieldeutig. In Zusammenhang mit anamnestischen Angaben sowie Lokalisation und Verteilung der Veränderungen lassen sich jedoch in vielen Fällen Hinweise zur Ätiologie ableiten. 


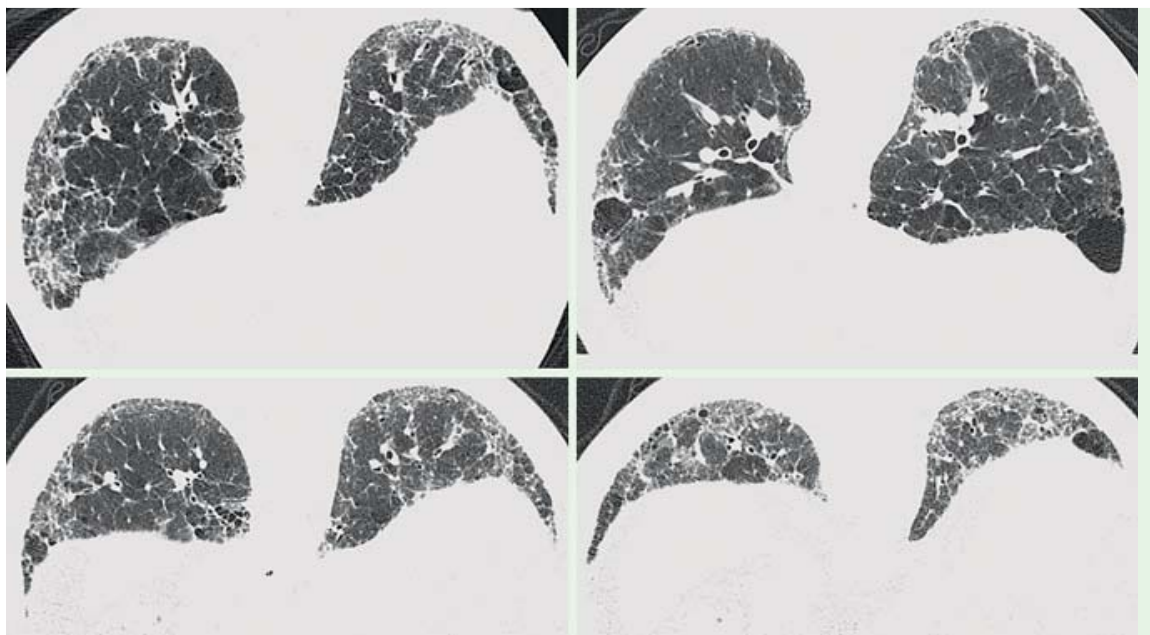

Abb. 1 Stark ausgeprägte Veränderungen bei Asbestose (die obige Fenstereinstellung dient nur zur besseren Demonstration der parenchymalen fortgeschrittenen Veränderungen; bei HRCT-Untersuchung unbedingt weites Fenster mit $\mathrm{W}=2000$ und $\mathrm{L}=-350$ bis $-500 \mathrm{HU}$ anwenden).
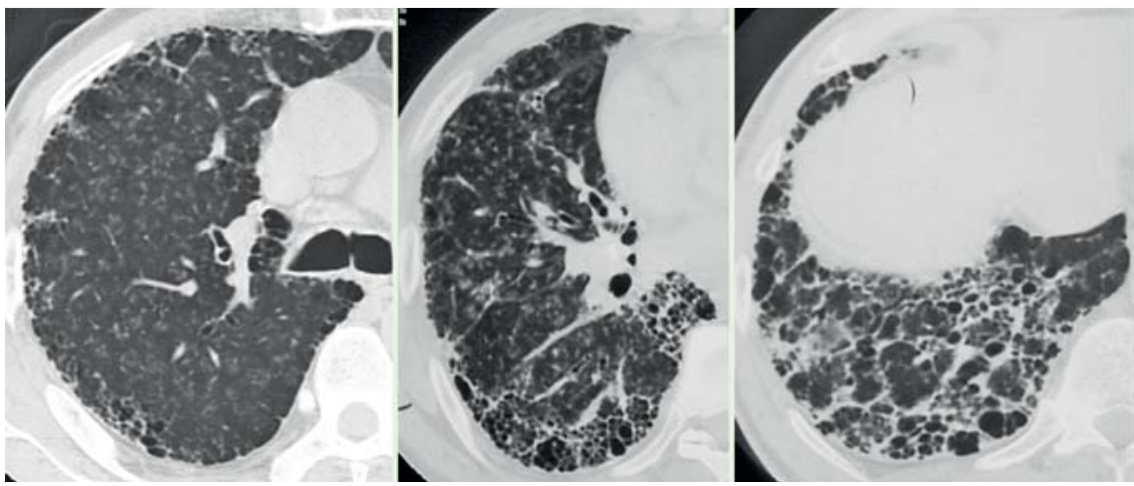

Abb. 2 Referenzfilme; Honeycombing Stadium I-III [2].

Asbestfaserstaubbedingte Veränderungen sind in der Regel dorsobasal betont. In frühen Stadien finden sich asbestfaserstaubbedingte Veränderungen bevorzugt subpleural bzw. im Bereich des Lungenmantels. Abb. 1 zeigt charakteristische, stark ausgeprägte Veränderungen einer Asbestose.

In fortgeschrittenen Fällen einer Asbestose kann es zu einem sog. Honeycombing mit wabenartigem Parenchymmuster kommen (ه Abb. 2).

Besonders schwierig $\mathrm{zu}$ interpretieren sind gering ausgeprägte, ausschließlich parenchymale Veränderungen, weil sie mit der geringsten Spezifität einhergehen ( $\bullet$ Abb. 3).

Grundsätzlich gilt, dass das gleichzeitige Vorhandensein charakteristischer pleuraler Verdickungen (s. u.) die ätiologische Zuordnung parenchymaler Veränderungen zu einer Asbestgenese wesentlich erleichtert ( $\bullet$ Abb. 4) [3].

In den abhängigen Partien der Lunge - vor allem dorsal subpleural - können intralobuläre fibrotische Veränderungen durch Hypostaseeffekte vorgetäuscht werden. In diesen Fällen ist eine ergänzende Untersuchung in Bauchlage zur Abklärung erforderlich. Bei Persistenz der Verdichtungen in Bauchlage ist von einer Fibrose auszugehen ( $\bullet$ Abb. 5).

\section{Pleurale Veränderungen nach Asbestexposition \\ $\nabla$}

Grundsätzlich können folgende pleurale Verdickungen mit einer Asbestexposition in Zusammenhang gebracht werden. Dabei ist von einer abnehmenden Spezifität für eine asbestinduzierte Genese von A-H auszugehen: a) Beidseitige verkalkte Pleuraplaques (parietal):

Tafelbergartig

Nicht tafelbergartig

b) Einseitige verkalkte Pleuraplaques (parietal):

Tafelbergartig

c) Beidseitige nicht verkalkte Pleuraplaques (parietal):

Tafelbergartig

Nicht tafelbergartig

d) Einseitige verkalkte Pleuraplaques (parietal):

Nicht tafelbergartig

e) Einseitig nicht verkalkte Pleuraplaques (parietal):

Tafelbergartig

Nicht tafelbergartig

f) Pleuraverdickungen (viszeral):

Verdickungen mit davon ausgehenden parenchymalen

Bändern/irregulären Schatten

„Hyalinosis complicata“ (Z.n. Pleuraerguss)

g) Rundatelektase

h) Pleuraerguss

Bezüglich der röntgenanatomischen Topografie asbestverursachter pleuraler Läsionen wird auf [5] verwiesen.

- Abb. 6 stellt mögliche Manifestationsformen bzw. Varianten einiger der genannten Veränderungen schematisch dar.

Nach der neuen Internationalen CT-Klassifikation wird bei den Manifestationsformen pleuraler Verdickungen primär zwischen

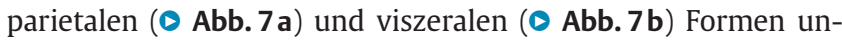
terschieden [2]. Dabei sind viszerale Formen durch parenchymale Bänder oder feine irreguläre Schatten charakterisiert, die von der pleuralen Verdickung ausgehend in das Parenchym einstrahlen. 

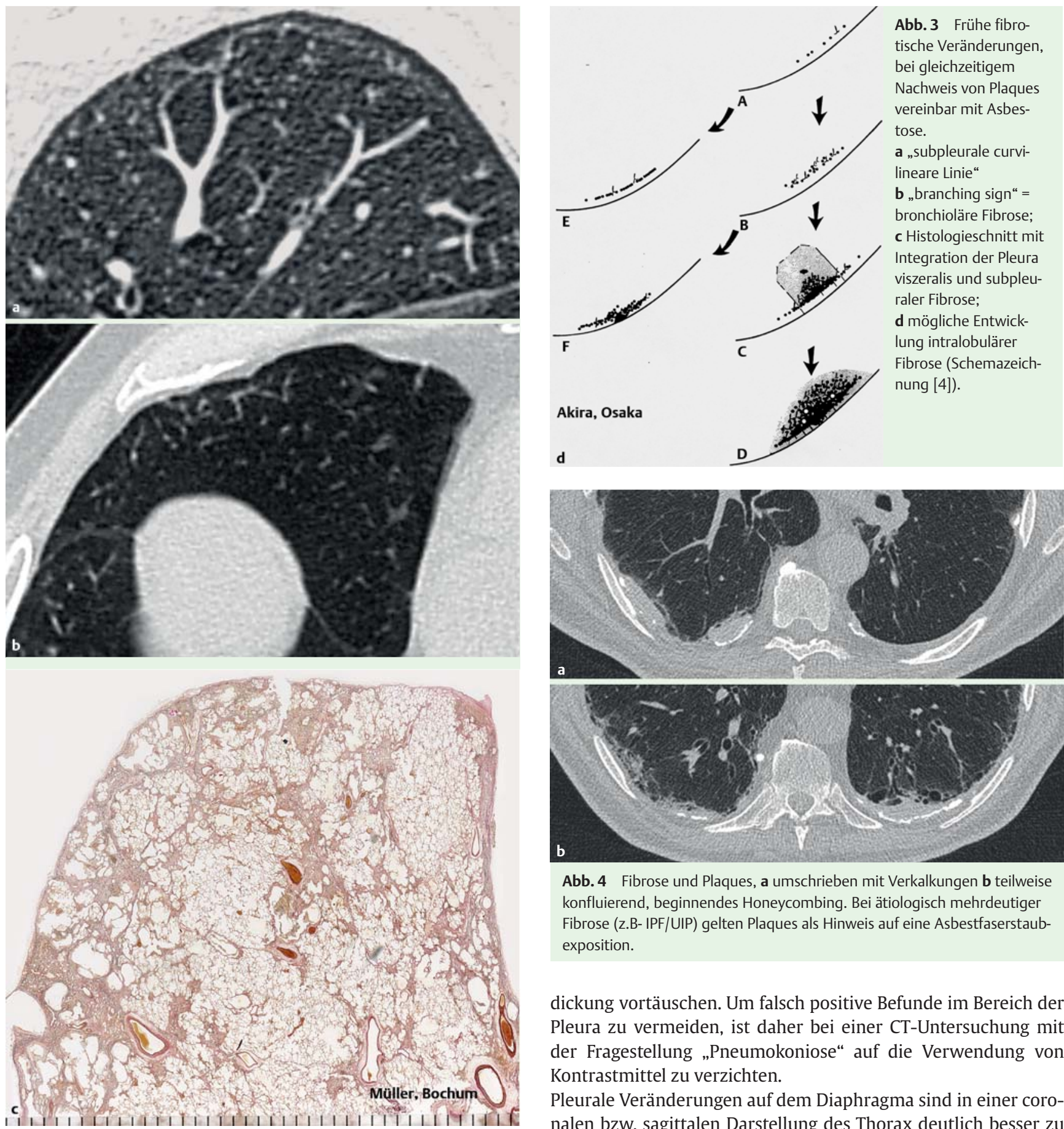

Abb. 4 Fibrose und Plaques, a umschrieben mit Verkalkungen $\mathbf{b}$ teilweise konfluierend, beginnendes Honeycombing. Bei ätiologisch mehrdeutiger Fibrose (z.B- IPF/UIP) gelten Plaques als Hinweis auf eine Asbestfaserstaubexposition.

dickung vortäuschen. Um falsch positive Befunde im Bereich der Pleura zu vermeiden, ist daher bei einer CT-Untersuchung mit der Fragestellung „Pneumokoniose“ auf die Verwendung von Kontrastmittel zu verzichten.

Pleurale Veränderungen auf dem Diaphragma sind in einer coronalen bzw. sagittalen Darstellung des Thorax deutlich besser zu erfassen $(\bullet$ Abb. 11). Daher ist eine Rekonstruktion der CT-Unter-

- Abb. 8 zeigt charakteristische parietale Plaques, z.T. mit Verkalkungen. Pleurale Verkalkungen sind erfahrungsgemäß bei einer asbestfaserstaubinduzierten Genese eher zentral innerhalb einer Plaque lokalisiert.

Schwierig zu interpretieren sind pleurale Verdickungen, die ohne charakteristische tafelbergartige Konfiguration imponieren, jedoch mit geringerer Spezifität ebenfalls asbestfaserstaubbedingt sein können. Dazu gehören zum Beispiel pleurale Verdickungen, die im Niveau der Pleura liegen und kurzstreckig oder längerstreckig verlaufen. Sie können unverkalkt oder verkalkt ( $\boldsymbol{Q}$ Abb. $9 \mathbf{b}$ ) und mit ( $\bullet$ Abb. 9a) extrapleuralem Fett oder ohne vorkommen. Eine Kontrastmittelgabe kann das Vorliegen pleuraler Verdickungen vortäuschen. $\bigcirc \mathbf{A b b} .10$ zeigt paravertebral beidseits Interkostalgefäße, die nach der Kontrastmittelgabe eine pleurale Versuchung in allen drei Raumebenen unbedingt empfehlenswert.

\section{Differenzialdiagnostische Überlegungen \\ $\nabla$}

Grundsätzlich können alle radiologisch fassbaren Veränderungen, die in Verbindung mit einer Exposition gegenüber Asbestfaserstaub gebracht werden, auch durch anderweitige Einflüsse verursacht werden. Kein röntgenmorphologischer Befund weist eine Spezifität von $100 \%$ für eine Asbestverursachung auf. Liegen ausschließlich parenchymale Veränderungen vor und finden sich keine Pleuraplaques, gelingt die Differenzialdiagnose zwischen einer idiopathischen Lungenfibrose und einer Asbestose aufgrund röntgenmorphologischer Kriterien alleine nicht hinreichend sicher. 


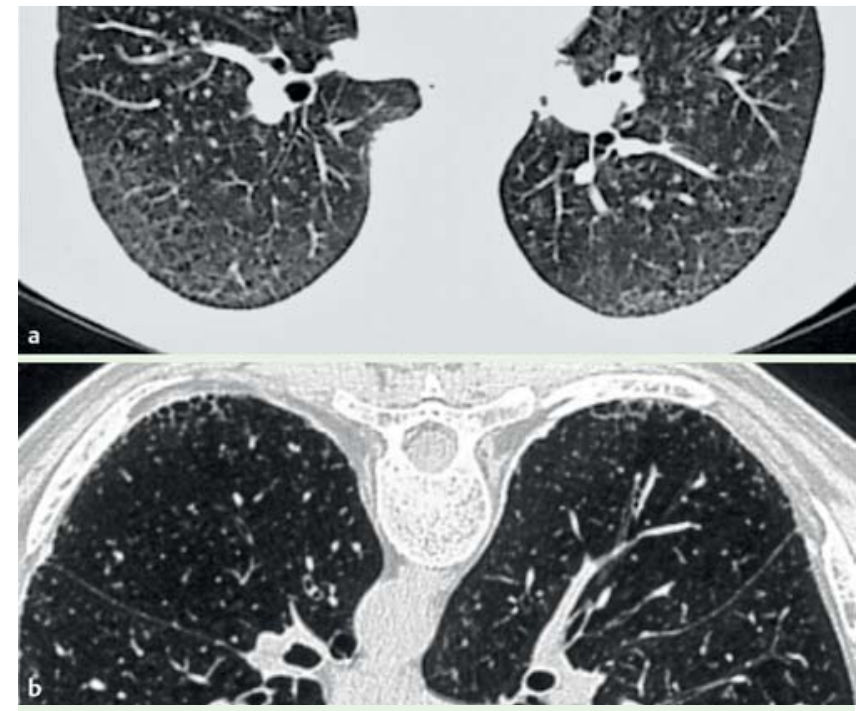

Abb. 5 Geringe Fibrose, a Rückenlage mit Hypostase, b Bauchlage mit geringen persistierenden intralobulären fibrotischen Veränderungen, die die Pleura viszeralis mit einbeziehen (ohne Nachweis von Plaques beginnende Asbestose möglich).

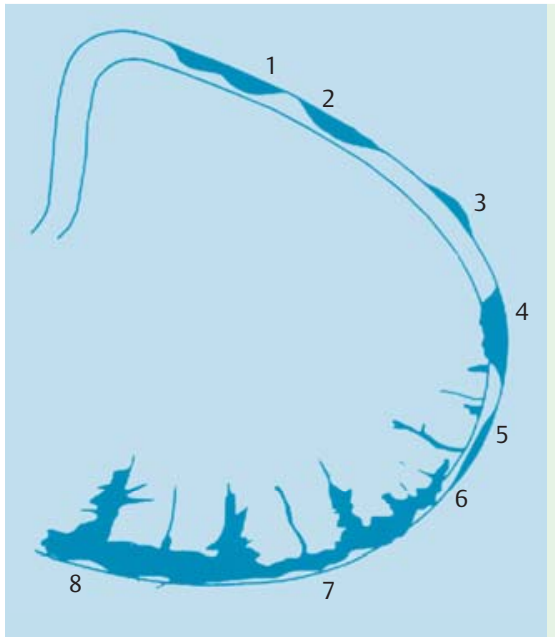

Abb. 6 Beispiele für pleurale Verdickungen im CT/HRCT.
1-3 = Plaquesformationen, charakteristisch für „parietale“ Form

1 = „Tafelberg“ mit steiler Kante

2 = flacher Randabfall

$3=$ flache, intercostale Entwicklung

$4=$ Plaque mit geringer intralobulärer Fibrose

5 = intercostale Plaque d. Pl. parietalis mit initialer Fibrose viszeral und subpleural, als viszerale Plaque kodiert

$6-8=$ Pleurafibrose mit Hyalinosis complicata, auch mit Rundatelektase möglich.
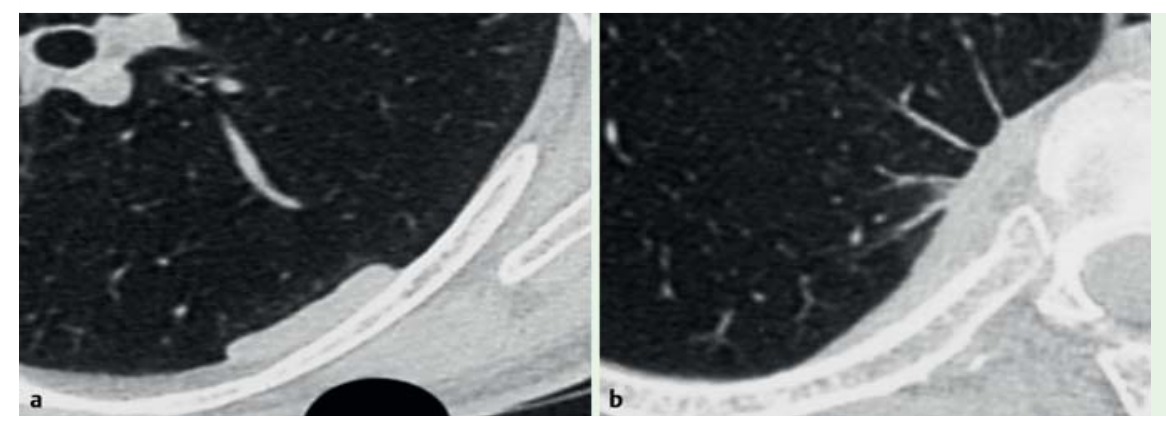

Abb. 7 Plaque: a „parietale“ Form (Tafelberg), b "viszerale“ Form.
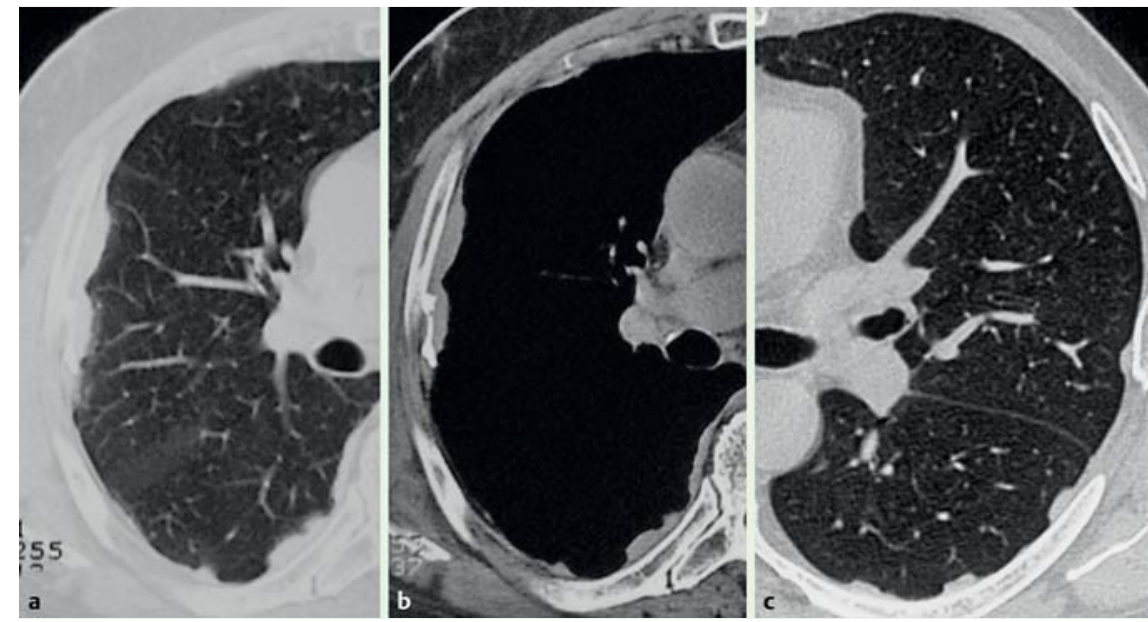

Eine gewisse Hilfestellung können die in $\bullet$ Tab. 1 aufgeführten röntgenmorphologischen Befunde bei der Differenzierung zwischen beiden Krankheitsentitäten liefern.

Umfassende Daten zur Prävalenz pleuraler Veränderungen bei beruflich nicht asbestexponierten Personengruppen liegen für konventionelle Röntgenaufnahmen vor ( Tab. 2). Bei der Interpretation dieser Daten ist zu berücksichtigen, dass die einzelnen Studien über einen Zeitraum von 60 Jahren mit deutlichen Veränderungen in der Technik und Qualität durchgeführt wurden und auch die Klassifikationskriterien in den Studien nicht einheitlich sind. Darüber hinaus ist die Sensitivität und Spezifität der konventionellen Röntgentechnik im Vergleich zur HRCT bekanntermaßen deutlich niedriger [6]. In den Studien zeigt sich eine Prävalenz pleuraler Plaques oder Verkalkungen mit einer Spannbreite von 0,02\% (USA) bis 12,8\% (Finnland), wobei in Finnland natürliche Faservorkommen für die hohen Zahlen verantwortlich gemacht werden. 


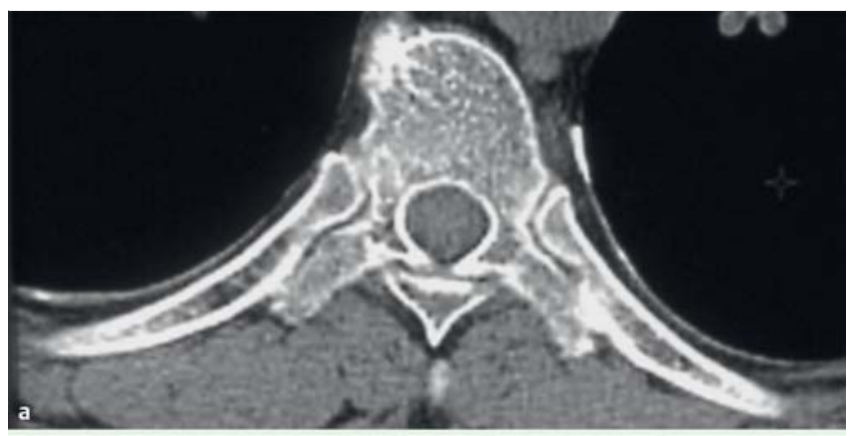

Tab. 1 Vergleich der Häufigkeit von HRCT-Befunden bei Asbestose und idiopathischer Lungenfibrose (IPF).

\begin{tabular}{|llll|}
\hline $\begin{array}{l}\text { HRCT Befund } \\
\text { Interlobuläre septale } \\
\text { Verdickung }\end{array}$ & $\begin{array}{l}\text { Asbestose (\%) } \\
\text { IPF (\%) }\end{array}$ & $\begin{array}{l}\text { p-Wert } \\
\text { NS }\end{array}$ \\
$\begin{array}{l}\text { Intralobuläre } \\
\text { Verschattungen }\end{array}$ & 69 & 98 & $<0.0001$ \\
\hline $\begin{array}{l}\text { Subpleurale „dots“| } \\
\text { branching sign }\end{array}$ & 81 & 25 & $<0.0001$ \\
\hline $\begin{array}{l}\text { Honeycombing } \\
\text { grobes honeycombing } \\
\text { (>5 mm) }\end{array}$ & 34 & 76 & $<0.0001$ \\
\hline $\begin{array}{l}\text { Traktionsbronchiek- } \\
\text { tasen }\end{array}$ & 69 & 35 & $<0.0001$ \\
\hline $\begin{array}{l}\text { Sichtbare intralobu- } \\
\text { läre Bronchiolen }\end{array}$ & 20 & 95 & $<0.0001$ \\
\hline $\begin{array}{l}\text { Subpleurale lineare } \\
\text { Verschattungen }\end{array}$ & 69 & 78 & $<0.0001$ \\
\hline $\begin{array}{l}\text { Parenchymale Bänder } \\
\text { Pleural plaques/ }\end{array}$ & 48 & 28 & $<0.0001$ \\
thickening & 83 & 4 & $<0.0001$ \\
\hline \begin{tabular}{l} 
Modifiziert nach [4] \\
\hline
\end{tabular} & 4 & $<0.0001$ \\
\hline
\end{tabular}

Abb. 9 Parietale Plaques, teils verkalkt, im Niveau der Pleura (nach HRCTSchema $=$ Dicke $\mathbf{a})(\mathbf{b}=$ unterhalb Plaque extrapleurales Fett $)$.

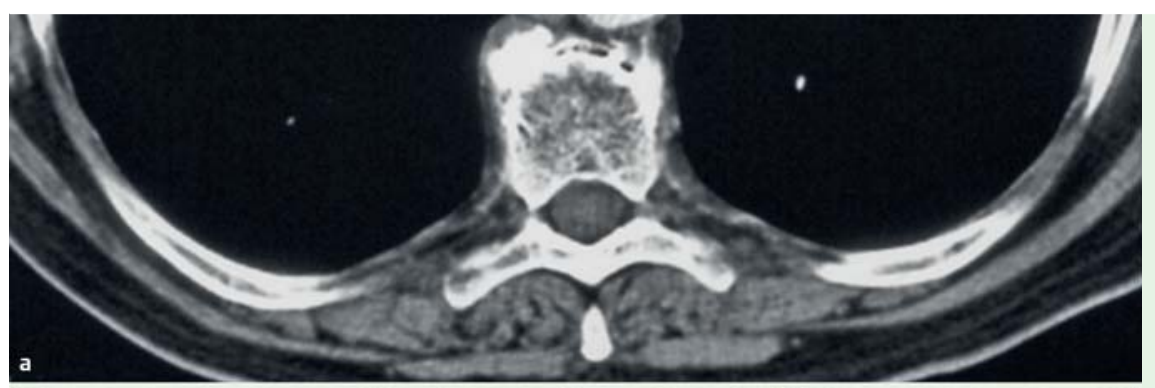

Abb. 10 Vortäuschung von Plaques durch Intercostalgefäße: $\mathbf{a}$ ohne $\mathbf{b}$ nach KM.
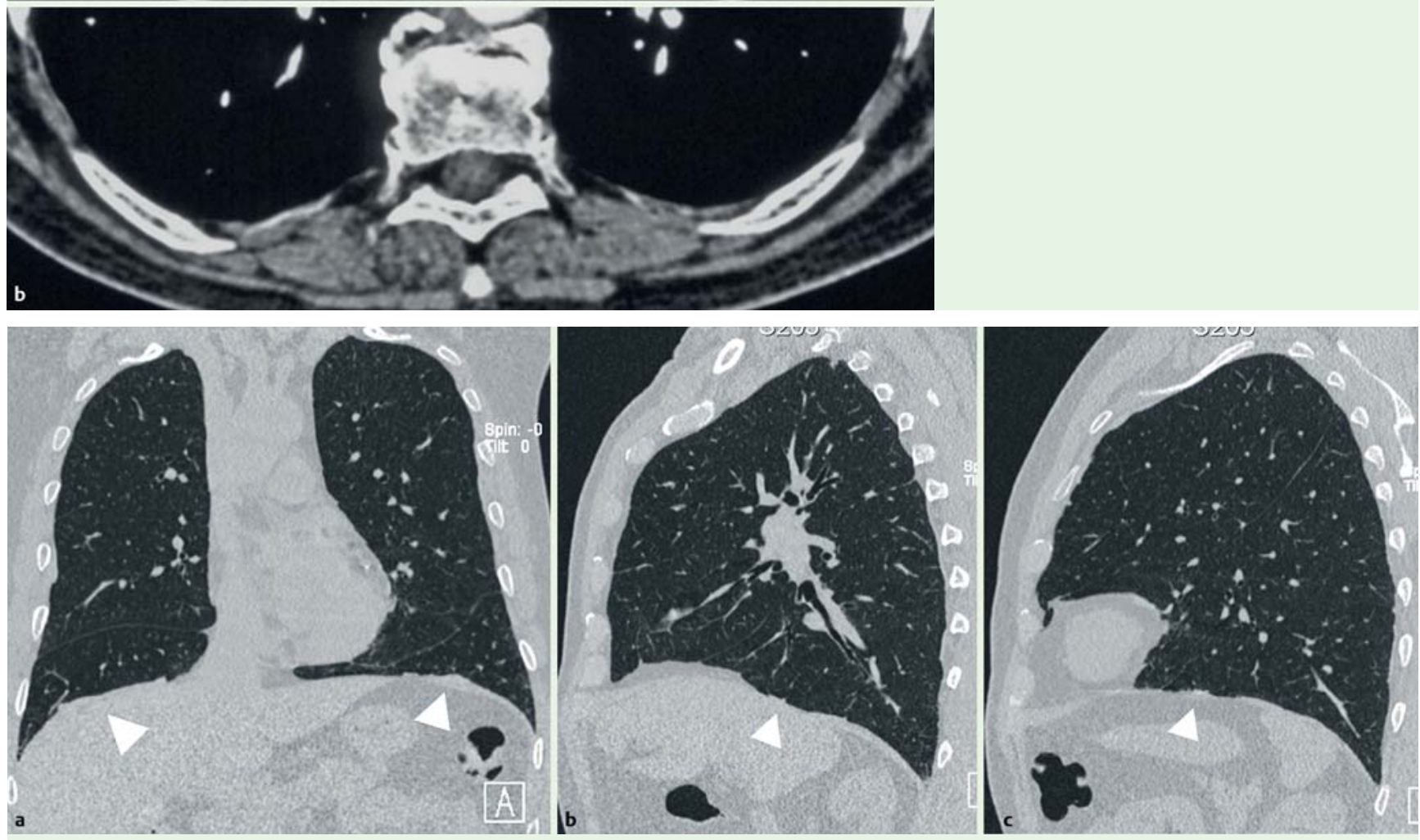

Abb. 11 a-c Beispiel für coronale (frontale) und sagittale Rekonstruktion zur Darstellung kleiner Plaques im Diaphragmabereich (Pfeile). 


\begin{tabular}{|c|c|c|c|}
\hline Studiengruppe & $\mathbf{N}$ & $\begin{array}{l}\text { Prävalenz pleuraler Plaques } \\
\text { oder Kalzifikationen (\%) }\end{array}$ & Literaturquelle \\
\hline \multicolumn{4}{|l|}{ USA } \\
\hline Allgemeine Bevölkerung & 115000 & $0,02^{2}$ & {$[8]$} \\
\hline \multicolumn{4}{|l|}{ Westdeutschland } \\
\hline Allgemeine Bevölkerung & 111069 & $0,03^{2}$ & [9] \\
\hline $\begin{array}{l}\text { Ostdeutschland } \\
\text { Ländliche Region }\end{array}$ & 87036 & $0,54^{3}$ & {$[10]$} \\
\hline Schweden, männlich über 40 Jahre & 33011 & $0,97^{3}$ & {$[10]$} \\
\hline \multicolumn{4}{|l|}{ Finnland } \\
\hline Allgemeine Bevölkerung & 35000 & $0,22^{2}$ & {$[11]$} \\
\hline $\begin{array}{l}\text { Frauen der Gruppe älter als } 30 \mathrm{~J} . \\
\text { (nur beidseitig) }^{1}\end{array}$ & 3815 & $1,9^{3}$ & {$[12]$} \\
\hline $\begin{array}{l}\text { Frauen der Gruppe älter als } 30 \mathrm{~J} . \\
\text { (beidseitig + einseitig) }^{1}\end{array}$ & 3815 & $3,4^{3}$ & [12] \\
\hline $\begin{array}{l}\text { Männer der Gruppe älter als } 30 \mathrm{~J} \text {. } \\
\text { (nur beidseitig) }^{1}\end{array}$ & 3280 & $7,0^{3}$ & {$[12]$} \\
\hline $\begin{array}{l}\text { Männer der Gruppe älter als } 30 \mathrm{~J} \text {. } \\
\text { (beidseitig + einseitig) }^{1}\end{array}$ & 3280 & $12,8^{3}$ & {$[12]$} \\
\hline \multicolumn{4}{|l|}{ Tschechoslowakei } \\
\hline Allgemeine Bevölkerung & 8133 & $0,34^{2}$ & [13] \\
\hline Allgemeine Bevölkerung & 44549 & $0,54^{2}$ & {$[14]$} \\
\hline Allgemeine Bevölkerung & 9871 & $2,77^{2}$ & {$[15]$} \\
\hline Allgemeine Bevölkerung & 9760 & $6,6^{2}$ & {$[15]$} \\
\hline \multicolumn{4}{|l|}{ Italien } \\
\hline Erwachsene & 4000 & $0,58^{2}$ & {$[16]$} \\
\hline
\end{tabular}

Tab. 2 Prävalenz pleuraler Plaques oder pleuraler Kalzifikationen auf konventionellen Röntgenthoraxaufnahmen in Personengruppen ohne dokumentierte Asbestexposition (modifiziert und ergänzt nach [7]).

${ }^{1}$ Mini Finland Health Umfrage. ${ }^{2}$ pleurale Kalizifikationen. ${ }^{3}$ pleurale Plaques.

\begin{tabular}{ll} 
Möglicher Einflussfaktor & Literaturquelle \\
Erionit & {$[18,19]$} \\
\hline Wollastonit & {$[20]$} \\
\hline Künstliche Glasfasern & {$[21-25]$} \\
\hline Kaolin & {$[26,27]$} \\
\hline Sepiolith & {$[28]$} \\
\hline Glimmer & {$[29,30]$} \\
\hline Tuberkulose & {$[8,31]$} \\
\hline Empyem & {$[8],[32-34]$} \\
\hline Pleuritis mit Erguss oder schwere Pleuritis* & {$[8,34,31,35]$} \\
\hline Bilaterale Pneumonien in Krankengeschichte & {$[33]$} \\
\hline Aktinomykose & {$[8]$} \\
\hline Hämothorax & {$[32,34,31]$} \\
\hline Rheumatoide Arthritis & {$[33,36,37]$} \\
\hline Sclerodermie & {$[35,38,39]$} \\
\hline Beidseitige pulmonale Embolie mit Erguss in Krankengeschichte & {$[33,35]$} \\
\hline Infektion (pyogen, tuberkulös oder pilzbedingt) & {$[35,38]$} \\
\hline Trauma & {$[8,35]$} \\
\hline Rippenfraktur & {$[33,38]$} \\
\hline Kalzifizierender, fibröser Pseudotumor der Pleura & {$[40]$} \\
\hline Chronische Mineralöl-Aspiration & {$[34,38]$} \\
\hline Ankylosierende Spondylitis & {$[41]$} \\
\hline Chronische Niereninsuffizienz & {$[33]$} \\
\hline Hämatogene Metastasen & {$[32]$} \\
\hline Hämorrhagie & {$[38]$} \\
\hline Vormalige Thoraxoperation & {$[33]$} \\
\hline Lokale neoplastische Invasion & {$[38]$} \\
\hline Pneumothorax & {$[32]$} \\
\hline Bestrahlung & {$[38]$} \\
\hline eine Plen & {$[3]$} \\
\hline
\end{tabular}

Tab. 3 Nicht asbestfaserstaubbezogene Faktoren, potenziell assoziiert mit pleuralen Plaques, Verdickungen und Kalzifikationen aus epidemiologischen Studien, Fallberichten und klinischen Reviews (modifiziert und ergänzt nach [7]).

* eine Pleuritis (mit/ohne Erguss kann i. S. einer Asbestpleuritis auch asbestverursacht auftreten.

Grundsätzlich müssen auch bei pleuralen Veränderungen neben einer Asbestfaserstaubgenese eine Vielzahl möglicher anderer Ursachen differenzialätiologisch in Betracht gezogen werden [17].
- Tab. 3 zeigt Ergebnisse aus epidemiologischen Studien, Übersichtsarbeiten und Fallberichten zu asbestfaserstaubunabhängigen Faktoren, die zu pleuralen Verdickungen führen können.

Bei der Beurteilung computertomografischer Aufnahmen und insbesondere bei der sich an den Befund anschließenden Kausal- 
zusammenhangsbeurteilung ist zu prüfen, inwiefern konkurrierende Faktoren für den parenchymalen oder pleuralen Befund ursächlich oder wesentlich teilursächlich sind (Theorie der wesentlichen Bedingung).

Dabei ist aber auch zu berücksichtigen, dass z. B. postspezifische Veränderungen nicht a priori asbestfaserstaubbedingte Veränderungen ausschließen. Sollte die CT-Untersuchung Anhaltspunkte dafür ergeben, dass die vorliegenden Veränderungen durch eine andersartige Erkrankung verursacht sein könnten, muss diese Vermutung durch weiterführende Ermittlungen oder Befunde belegt werden. Der bloße Verdacht auf das Vorliegen asbestunabhängiger Befunde reicht zum Ausschluss einer BK nicht aus.

\section{Fazit}

\section{$\nabla$}

Der Wahrscheinlichkeitsbeweis asbestbedingter Veränderungen kann nur in Zusammenschau von Informationen zur Anamnese, Berufsanamnese sowie zu bildgebenden und/oder histologischen Befunden geführt werden. Die dargestellten Beispiele sollen als Hilfestellung bei der Kausalzusammenhangsbeurteilung dienen. Ergebnisse aktuell laufender wissenschaftlicher Studien können in den nächsten Jahren zu einer Präzisierung von Empfehlungen für eine BK-Anzeige bzw. für die Bejahung eines Kausalzusammenhangs zwischen einer beruflichen Asbestexposition und dem CT-morphologischen Bild führen. Insofern sind die hier dargestellten Erkenntnisse als vorläufig anzusehen und im Rahmen wissenschaftlicher Studien weiterzuentwickeln.

\section{Interessenkonflikte}

$\nabla$

Die Autoren geben an, dass kein Interessenkonflikt besteht.

\author{
Institute \\ ${ }^{1}$ Institut für Arbeitsmedizin und Sozialmedizin, Universitätsklinikum \\ RWTH Aachen University \\ 2 Institut für Arbeitsmedizin der Heinrich-Heine-Universität Düsseldorf \\ 3 BGBAU, AMD Hannover \\ 4 BGBAU, AMD Karlsruhe \\ Knappschaftskrankenhaus Dortmund \\ Evangelisches Lungenkrankenhaus Berlin-Buch \\ BDT Institut für bildgebende Diagnostik \& Therapie Erlangen \\ 8 Neuruppin \\ ${ }^{9}$ Hermannsburg \\ 10 BG-Klinik für Berufskrankheiten Falkenstein \\ 11 GVS - Gesundheitsvorsorge (vormals: ZAs), Berufsgenossenschaft \\ Energie Textil Elektro, Augsburg \\ 12 DGUV, Sankt Augustin \\ ${ }^{13}$ Klinik für Berufskrankheiten Bad Reichenhall \\ 14 Institut für Arbeits-, Sozial- und Umweltmedizin der Universität \\ Erlangen-Nürnberg \\ ${ }^{15}$ Berlin \\ 16 Walldorf
}

\section{Literatur}

1 Suganuma N, Kusaka Y, Hering KG et al. Reliability of the Proposed International Classification of High-Resolution Computed Tomography for Occupational and Environmental Respiratory Diseases. J Occup Health 2009; 17: [Epub ahead of print]

2 Hering KG, Tuengerthal S, Kraus T. Standardisierte CT/HRCT-Klassifikation der Bundesrepublik Deutschland für arbeits- und umweltbedingte Thoraxerkrankungen. Der Radiologe 2004; 44: 500-511

3 Webb WR, Müller NL, Naidich DP. High Resolution of the Lung. Philadelphia: Wolters Kluwer Lippincott Williams \& Wilkins, 2009: 301ff.
4 Akira M, Yamamoto S, Inoue $Y$ et al. High-resolution CT of asbestosis and idiopathic pulmonary fibrosis. Am J Roentgenol 2003; 181: $163-$ 169

5 Kraus T, Borsch-Galetke E, Elliehausen HJ et al. Anzeigekriterien asbestfaserstaubbedingter Erkrankungen gemäß BK-Nr. 4103 BKV. Pneumologie 2009; 63: 726-732

6 Kraus T, Raithel HJ. Frühdiagnostik Asbest-verursachter Erkrankungen - Differenzierte Vorsorgestrategie bei Asbeststaubexposition - Arbeitsmedizinische Längsschnittuntersuchungen bei einem Hochrisikokollektiv ehemals asbeststaubexponierter Arbeitnehmer; Hauptverband der gewerblichen Berufsgenossenschaften, St. Augustin, 1998

7 Clarke CC, Mowat FS, Kelsh MA, Roberts MA. Pleural plaques: a review of diagnostic issues and possible nonasbestos factors. Arch Environ Occup Health 2006; 61: $183-192$

8 Floyd C, Hapburn R. Calcification of the pleura. Am Rev Tuberculosis 1939; 40: 215-223

9 Neef W. Beidseitige Pleuraverkalkungen im Alter. Fortschr Geb Röntgenstr Nuklearmed 1963; 99: 632-638

10 Löhr J, Klippe HJ, Kroeger C. Surgical treatment of diffuse mesothelioma of the pleura; curative and palliative possibilities. Prax Klin Pneumol 1981; 35: $394-399$

11 Kiviluoto R. Pleural calcification as a roentgenologic sign of non-occupational endemic anthophyllite-asbestosis. Acta Radiol 1960; 194 (suppl): 1-67

12 Zitting AJ. Prevalence of radiographic small lung opacities and pleural abnormalities in a representative adult population sample. Chest 1995; 107: $126-131$

13 Navratil M, Trippe F. Prevalence of pleural calcification in persons exposed to asbestos dust, and in the general population in the same district. Environ Res 1972; 5: 210-216

14 Marsova D. Beitrag zur Ätiologie der Pleuraverkalkungen. Z Tuberk 1964; 121: 329-334

15 Hromek J. The mass incidence of characteristic pleural changes in citizens of the wester part of the former Juhlava region. Rozhl Tuberk 1962; 22: 405-415

16 Miraglia E, Meola G. Contributo allo delle alcificatzioni pleuriche. Arch Tisiol Mal Appar Respir 1960; 15: 1005-1023

17 Müller KM. Pleura: Pathologie nicht-neoplastischer Erkrankungen. Pneumologie 2004; 58: 516 - 524

18 Baris I, Simonato L, Artvinli M et al. Epidemiological and environmental evidence of the health effects of exposure to erionite fibres: a four-year study in the Cappadocian region of Turkey. Int J Cancer 1987; 39: 10 17

19 Karakoca Y. Environmental pleural plaques due to asbestos and fibrous zeolite exposure in Turkey. In: Hoskins JA, Krummer HW, Soler M, eds Pathogenesis. Diagnosis an Clinical Relevance of Pleural Plaques. New York: Karger, 1997

20 Huuskonen MS, Tossavainen A, Koskinen $\mathrm{H}$ et al. Wollastonite exposure and lung fibrosis. Environ Res 1983; 30: 291 - 304

21 Weill H, Hughes JM, Hammad YY et al. Respiratory health in workers exposed to man-made vitreous fibers. Am Rev Respir Dis 1983; 128: $104-112$

22 Järvholm B, Hillerdal G, Järliden AK et al. Occurrence of pleural plaques in workers with exposure to mineral wool. Int Arch Occup Environ Health 1995; 67: 343-346

23 Lockey J, Lemasters GK, Rice C et al. Refractory ceramic fiber exposure and pleural plaques. Am J Respir Crit Care Med 1996; 154: 1405-1410

24 Lockey J, Lemasters GK, Levin L et al. A longitudinal study of chest radiographic changes of workers in the refractory ceramic fiber industry. Chest 2002; 121: 2044-2051

25 Cowie HA, Wild P, Beck J et al. An epidemiological study of the respiratory health of workers in the European refractory ceramic fibre industry. Occup Environ Med 2001; 58: 800 - 810

26 Chaudhary BA, Kanes GJ, Pool WH. Pleural thickening in mild kaolinosis. South Med J 1997; 90: 1106-1109

27 Gough J, Hale LW, King EJ et al. Pneumoconiosis of kaolin workers. Br J Ind Med 1956; 13: $251-259$

28 McConnochie K, Bevan C, Newcombe RG et al. A study of Spanish sepiolite workers. Thorax 1993; 48: 370-374

29 Kleinfeld M. Pleural calcification as a sign of silicatosis. Am J Med Sci 1966; 251: $215-224$

30 Skulberg KR, Gylseth B, Skaug V, Hanoa R. Mica pneumoconiosis - a literature review. Scand J Work Environ Health 1985; 11: 65 - 74

31 Head J. Calcification of the pleura. Ann Int Med 1934; 7: 1295-1307 
32 Anderson HA, Selikoff U. Pleural reactions to environmental agents. Fed Proc 1978; 37: $2496-2500$

33 Albelda SM, Epstein DM, Gefter WB, Miller WT. Pleural thickening: its significance and relationship to asbestos dust exposure. Am Rev Respir Dis 1982; 126: $621-624$

34 Felson B. Chest Roentgenology. Philadelphia, PA: WB Saunders, 1973: 489-492

35 Romey R, Moskowitz M. Scleroderma, pleural calcification and reticulum cell sarcoma of the lungs. Chest 1973; 64: 371- 373

36 Talbott JA, Calkins E. Pulmonary Involvement In Rheumatoid Arthritis. JAMA 1964; 189: 911 -913
37 Remy-Jardin M, Remy J, Cortet B et al. Lung changes in rheumatoid arthritis: CT findings. Radiology 1994; 193: 375 - 382

38 Sargent EN, Jacobson G, Gordonson JS. Pleural plaques: a signpost of asbestos dust inhalation. Semin Roentgenol 1977; 12: 287-297

39 Weaver AL, Divertie MB, Titus JL. Pulmonary scleroderma. Dis Chest 1968; 54: $490-498$

40 Pinkard NB, Wilson RW, Lawless $N$ et al. Calcifying fibrous pseudotumor of pleura. A report of three cases of a newly described entity involving the pleura. Am J Clin Pathol 1996; 105: 189-194

41 Crompton GK, Cameron SJ, Langlands AO. Pulmonary fibrosis, pulmonary tuberculosis and ankylosing spondylitis. Br J Dis Chest 1974; 68: $51-56$ 